\title{
Sind wir schon (wieder) zu spät?
}

Ernst Gähler ${ }^{a}$, Anton Prantl ${ }^{b}$, Franziska Zoggc, Ueli Zihlmannd, Urs Stoffele

a Dr. med., Vizepräsident FMH, Verantwortlicher Ressort Tarife und Verträge

b Direktionspräsident Ärztekasse

c Vorstandsmitglied AGZG, Hausärzte $\mathrm{CH}$, VZAG

d Geschäftsführer Medkey

e Co-Präsident KKA

\section{Ausgangslage}

Immer wieder ist die Ärzteschaft dem Vorwurf ausgesetzt, für die Mengenausweitung im Gesundheitswesen verantwortlich zu sein. Dies ohne stichhaltige Argumente, denn es fehlt in allen Statistiken eine Klassierung der Nachfrage nach medizinischen Leistungen.

Die Demographie, neue Versorgungsmodelle, aber auch behördliche Verordnungen - denken wir etwa an Impfungen, aber auch an die leidige Revision der Analysenliste - haben teilweise grosse Auswirkungen auf die Nachfrage nach Leistungen. Die Einführung der Fallpauschalen SwissDRG, im stationären Bereich per 1. Januar 2012 wird Nachfrageströme verschieben, sei es durch ausgelagerte Vorabklärungen und Nachbehandlungen, oder Selektion.

In den Spitälern einiger Kantone laufen seit einiger Zeit bereits Pilotprojekte nach AP-DRG, um sich auf den SwissDRG vorzubereiten und Erfahrungen zu sammeln, wie die Strukturen bis zur Einführung der Schweizerischen Fallpauschalen angepasst werden müssen. DRG wird also nicht erst ab 2012 eingeführt, sondern ist bereits Realität mit entsprechenden Konsequenzen.

Sind wir Ärzte also schon wieder zu spät? Wir brauchen verlässliche Grundlagen, um proaktiv und mit gutem Datenmaterial zu argumentieren. Die Turbulenzen rund um die Kostenverlagerung, welche die Einführung der DRG ganz sicher auslösen wird, sind bereits spürbar.

Erinnern wir uns an die Kostenneutralität bei der Einführung von TARMED. Wer die Mengenentwicklung und Leistung nicht begründen konnte, an dem blieben diese kompensationslos als Mengenausweitung «hängen»!

Beispiele zu DRG aus anderen Ländern, an denen wir uns orientieren könnten, z. B. aus Deutschland, gibt es nicht, denn da fehlen Daten aus dem ambulanten freiberuflichen Segment vor DRG gänzlich. Leider hat es Deutschland verpasst, die Situation vor der Einführung der Fallpauschalen abzubilden - diesen Fehler bedauern die Verantwortlichen ausserordentlich. Ähnliches wollen wir verhindern! Deshalb müssen wir schnell verlässliche Informationen und Daten zur Nachfrage erfassen.

\section{Das Projekt RFE}

Aus diesen Gründen ergriff die Zuger Ärztegesellschaft die Initiative und suchte zusammen mit den Partnern medkey (TrustCenter) und Ärztekasse nach einer Lösung, um eine solche Erhebung möglichst rasch, einfach und unkompliziert umzusetzen.
Mit dem Datenmonitoring Reason For Encounter RFE (zu Deutsch: «Grund für die Begegnung») soll jede Konsultation entsprechend klassiert werden. Die Erfassungsmethode und Terminologie sowie die Klassifizierung im RFE haben dann auf Empfehlung der Präsidentenkonferenz der VZAG (Vereinigung Zentralschweizer Ärztegesellschaften) alle Kantone der Zentralschweiz übernommen. Ausschlaggebend für das Datenmonitoring waren folgende Punkte:

- Erfassung muss für die Ärztin, den Arzt ohne wesentlichen Zusatzaufwand möglich sein;

- Leichte Verständlichkeit und Identifikation des Codes;

- Erhebung ist für den Arzt ohne Kostenfolge;

- Keine Änderung an bestehenden Strukturen und Standards (XML, Tarifpositionen, Diagnosen);

- Einfache Implementation für die Softwarehäuser minimaler Aufwand;

- Rasche Umsetzbarkeit ohne Verzögerung durch «pseudowissenschaftliche» Evaluationen.

\section{RFE und Dinamo}

RFE will sich nicht mit den Ansprüchen eines Dinamo messen. Ziel ist eine unverzügliche Umsetzung, ein einfaches, leicht anzuwendendes und schnell erhobenes Codesystem, das via das TrustCenter-System ohne Zusatzaufwand gesammelt werden kann.

Das RFE setzt bei einer einfachen Anwendung an: - Sehr beschränkte Anzahl Codes, die man sich leicht merken kann;

- Die Codes sind so, dass sie auch problemlos von der MPA gesetzt werden können;

- Wir haben die Softwarehäuser gebeten, RFE auch mit dem «Hamster» erfassbar zu machen.

\section{RFE und ICPC-2}

RFE hat sich als geeignetste Bezeichnung für den Anlass einer Konsultation erwiesen. Wir möchten allerdings Verwechslungen unbedingt vermeiden. RFE in diesem Projekt bezieht sich auf die Instanz, welche die Konsultation auslöst als Bestandteil der Leistungserfassung. Bei RFE im ICPC-2 stehen die Beschwerden der Patienten und die Prozedur im Zentrum. Dies ist Teil der elektronischen KG. Deshalb unsere Bitte: Das eine tun und das andere nicht lassen!

\section{Konsultation klassieren hilft Behauptungen entkräften}

Geplant ist, das Monitoring über mehrere Jahre laufen zu lassen und auf diese Weise Behauptungen mit einer soliden Datengrundlage zu entkräften. Die Einführung 
von SwissDRG ist Auslöser des Projekts RFE, aber keineswegs einziger Zweck. RFE wird auch dazu dienen, Daten zur Diskussion um die Mengenausweitung zu sammeln. Klassifiziert wird jede Konsultation mit einem entsprechenden RFE-Code. Mit der Rechnungsüberweisung an das TrustCenter wird dieser Code als Statistik-Argument mitgeliefert und ausgewertet. Folgende Zustände sind momentan codiert:

01 - Kontakt auf Wunsch des Patienten (Selbstzuweisung)
02 - Notfallkontakt
03 - Kontakt durch ärztliche Zuweisung
04 - Folgekontakt auf Verordnung des behandelnden
Arztes
05 - Folgekontakt wegen auswärtiger Hämatologie und
Chemie
06 - Kontakt in Zusammenhang mit Langzeitpflege
07 - Kontakt in kausalem Zusammenhang mit Eingriff/
Hospitalisation
99 - Kein Arztkontakt

\section{Eingabe der RFE-Codes}

Die Eingabe des RFE-Codes erfolgt bei der Leistungserfassung über eine zusätzliche Eingabemaske in der Praxissoftware. Da es sich um eine menugeführte Eingabe handelt, die einen zweistelligen Zahlencode zeigt, ist der Zusatzaufwand äusserst gering.

\section{RFE und Softwareanbieter für Ärzte}

Als es um die Implementierung dieses für die Ärzteschaft wichtigen Moduls ging, zeigten vor allem die grossen Software-Anbieter wie Vitodata und die Ärztekasse von Anfang an Interesse und grosse Kooperationsbereitschaft, so dass bereits nach wenigen Wochen RFE-Codes erfasst werden konnten. Die Entwicklung und Implementation des RFE-Modules wurde ohne Kostenfolge für die Ärztinnen und Ärzte umgesetzt.

Zurzeit bieten nebst Vitodata und der Ärztekasse auch Variosoft, TMR, Triamun und Advanced Concept entsprechende Software, RFE-Codes zu erfassen.

Machen Sie mit bei der RFE-Datenerfassung! Beginnen Sie noch heute mit der Erfassung des Besuchsgrundes Ihrer Patienten nach RFE. Voraussetzung: Ihre Praxissoftware hat entsprechendes Modul bereits freigeschaltet.

Helfen Sie mit bei der Verbreitung und bei der Erhebung der RFE-Codes: ein minimaler Aufwand für den Einzelnen, ein maximaler Effekt für alle. RFE ist für jedermann offen und lizenzfrei zu verwenden. Das bisherige Echo ausserhalb der Zentralschweiz ist überwältigend. Umso wichtiger ist es nun, das Projekt fortzuführen und zu verbreiten. Die Koordination und die Weiterentwicklung des Systems werden von medkey übernommen; das Ressort Tarife und Verträge der FMH, die KKA und die Hausärzte Schweiz unterstützen dieses Projekt voll und ganz. Ihre Anregungen und Fragen sind herzlich willkommen, denn RFE soll sich weiterentwickeln. Mehr Informationen finden Sie auf: www.aerzte-zs.ch/rfe.
Damit hat bereits ein grosser Teil der Ärzteschaft die Voraussetzungen, um am RFE-Datenmonitoring teilzunehmen.

Eine aktuelle Liste mit Details und einer Kurzanleitung zu RFE findet sich unter www.aerzte-zs.ch/rfe.

\section{Ihre Teilnahme erhöht die Aussagekraft}

Ungerechtfertigte Vorwürfe und Behauptungen können nur mit aussagekräftigen Daten widerlegt werden. Machen Sie mit bei der RFE-Datenerfassung: Ihre Teilnahme am RFE-Datenmonitoring leistet einen wichtigen Beitrag für eine solide Datengrundlage im Vorfeld zur Einführung der SwissDRG.

\section{RFE-Codes im Detail}

Eine Sitzung sollte mit einem RFE-Code versehen sein. Bei Mehrfachbelegung wird der Sitzung in der Auswertung der numerisch tiefste Wert zugewiesen. Die Codeliste in Tabelle 1 zeigt den Stand vom 1. Januar 2010. Sie soll und kann erweitert werden, aber mit Bedacht und bitte in Rücksprache mit den Initianten.

\section{Tabelle 1}

Liste mit RFE-Codes. Stand 1. Januar 2010.

\section{Kontakt auf Wunsch des Patienten}

02 Notfallkonsultation

03 Kontakt auf Zuweisung
04 Kontakt auf Verordnung /
Empfehlung

Dieser Code wird immer dann gesetzt, wenn der Kontakt vom Patienten oder seinen Angehörigen, allenfalls von Dritten initiiert wurde.

Bitte diesen Code ausschliesslich für Notfallkontakte im engeren Sinne $(00.2505$ bis 00.2590$)$ verwenden.

Kontakt auf eigene Empfehlung. Der zeitliche Abstand spielt keine Rolle. Verlaufskontrollen bei akuten oder chronischen Erkrankungen, Tumornachsorge, vereinbarte Vorsorgeuntersuchung usw.

05 Folgekontakt wegen auswärtiger Hämatologie oder Chemie

06 Kontakt im Zusammenhang mit Langzeitpflege

07 Kontakt in kausalem Zusammenhang mit Eingriff/Hospitalisation

Ausschlaggebend ist der Zusammenhang mit dem Eingriff oder der Hospitalisation - vorher oder nachher, unabhängig davon, wer die Konsultation ausgelöst hat (z. B. Aufklärungsgespräch vor Eingriff, präoperativer Untersuch, Nachbetreuung).

99 Kein Arztkontakt der Vollständigkeit. Kommt bei Laborsitzungen und/ oder Medikamentenbezug zum Tragen. 